\title{
An Interaction Torque Control Improving Human Force Estimation of the Rehab-Exos Exoskeleton
}

\author{
Massimiliano Solazzi ${ }^{1 *}$, Mirko Abbrescia ${ }^{2}$, Rocco Vertechy ${ }^{1}$, \\ Claudio Loconsole ${ }^{1}$, Vitoantonio Bevilacqua ${ }^{2}$, Antonio Frisoli ${ }^{1}$ \\ ${ }^{1}$ PERCRO Laboratory, TeCIP Institute, Scuola Superiore \\ Sant'Anna, Pisa \\ 2 Department of Electrical and Information Engineering, \\ Polytechnic University of Bari
}

\begin{abstract}
This paper describes the interaction torque control of the RehabExos, an upper-limb robotic exoskeleton with direct torque joint sensors for interaction in Virtual Environments and rehabilitation. The control architecture consists in a centralized torque control and separated optimal torque observers for each joint of the exoskeleton. The optimal observer is a full-state Kalman filter providing the estimates of both internal and external torques acting on the joints and overcoming most of the issues due to the noise in the torque sensor signals. The centralized torque control is based on a full dynamics model of the exoskeleton, calculates the kinematics and dynamics of the system and estimates the feed-forward contribution for the compensation of dynamic loads measured by joint torque sensors. Experimental tests have been carried out to validate the desired torque tracking in haptic interaction tasks.
\end{abstract}

Index Terms: H.5.2 [Information Interfaces and Presentation (HCI)]: User Interfaces-Haptic I/O; I.2.9 [Artificial intelligence]: Robotics-Kinematics and dynamics

\section{INTRODUCTION}

The robotic interfaces for physical human-robot interaction represent an important aspect of telexistence cockpits [2] and for interaction in Virtual Environments. They can come in different embodiments and realizations, but the exoskeleton represents the robotic system where the highest physical symbiosis with the human operator is achieved. Active exoskeleton systems are robotic devices that can be worn on the user's body, implying that they should satisfy requirements of safety and better compliance. After the Fukushima event in Japan, the application of these human-robot interfaces in the area of rescue robotics and teleoperation has became an emerging field of research, for which the development of upper limb active exoskeletons with dexterous manipulation abilities has become a hot topic of research. Another relevant sector of application of active exoskeletons is represented by neuro-motor rehabilitation poststroke [10], where different prototypes and commercial solutions have been recently proposed.

As shown in figure 1, exoskeletons built for rehabilitation and human power augmentation make use of different actuation solutions, such as geared solutions $[13,17,3]$, tendon drives $[6,15]$, hybrid solutions (screw and cable actuators) [7],variable-impedance actuators or even pneumatic actuation [16,9]. Recently also pure tendon solutions have been proposed with the Carex Exoskeleton [11]. For instance, both Berkeley Lower Extremity Exoskeleton (BLEEX) [8] and the XOS exoskeleton from Sarcos Research Corporation are based on hydraulic actuation systems, with control algorithms based on force measurements for a smooth control

*e-mail:m.solazzi@sssup.it

IEEE Haptics Symposium 2014

23-26 February, Houston, Tx, USA

978-1-4799-3131-6/14/\$31.00 @2014 IEEE

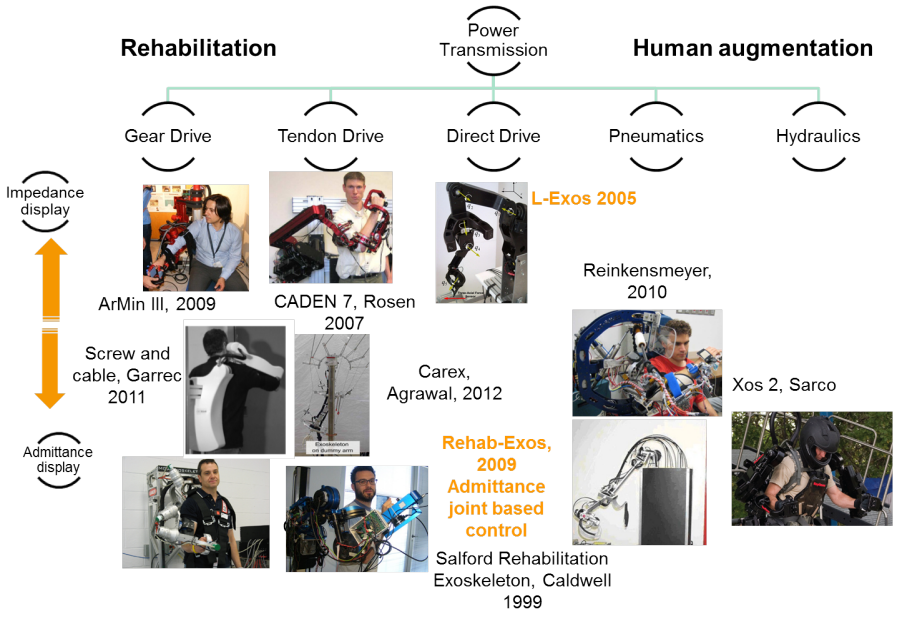

Figure 1: Recent solutions in upper limb exoskeleton design

of exoskeleton movement, while the Robot Suit HAL-5 (CYBERDYNE Inc., Japan) and the Body Extender [12] use electrical motors. Based on the adopted actuation, active exoskeletons can be classified as impedance based design (open-loop impedance control and impedance control with force feedback) or admittance-based design (admittance control with position feedback) [4], as outlined in figure 1.

Open-loop impedance control exoskeletons rely on lightweight designs with joint-delocated motors and backdrivable mechanical solutions, typically implemented making use of tendon transmissions [6], [15]; the most challenging limitations are the friction effects due to the transmission system, that can be compensated only by means of feed-forward compensation based on approximate models, the complexity of the transmission and the difficulty to be mechanically configured in a bilateral configuration, working both for left and right arm. On the other side admittance-based design requires force sensing and can achieve higher stiffness values, but relies on the adopted control for canceling system dynamics and inertia. Exoskeleton with a single force/torque sensor localization, such as one torque sensor at the exoskeleton elbow joint and a sixaxis force/torque sensor at the exoskeleton handle [5] or shoulder [14], can accurately regulate interaction forces at the exoskeleton terminal link (i.e. the handle) only. Lightweight robots with joint torque sensors [1] allow for multi-contact force/torque control (i.e. the regulation of interaction force/torques at multiple points distributed over multiple links).

The correct estimation of interaction force between human and exoskeleton in admittance designs requires control approaches that compensate for friction, inertial and gravity properties of the exoskeleton mechanical structure, but a complete cancellation of these 
effects is difficult to achieve. In this paper we present an interaction torque control of an exoskeleton with torque joint sensors for haptic rendering and rehabilitation purposes. With respect to previous work [18], we extend our control to take into account the multi-dof non linear system dynamics and provide a compensation of nonlinear effects such as inertial and gravity components, to achieve an accurate estimation of human interaction force. This is accomplished by a single joint optimum observer that ensures joint torque tracking, while a centralized control estimates and compensates for the dynamics of the whole system. The exoskeleton is endowed with a Force/Torque sensor at the handle to allow experimental evaluation on torque tracking performance and haptic interaction, that is presented in this work.

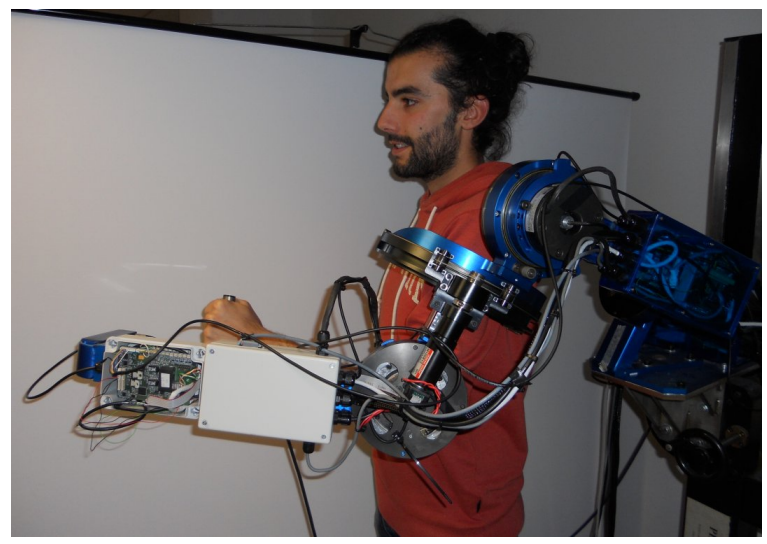

Figure 2: The Rehab-Exos exoskeleton

\section{The Rehab-Exos}

The Rehab-Exos is a active robotic exoskeleton (figure 2) conceived for rehabilitation applications. It is aimed at generating controlled contact forces/torques not only at its end-link handle, but also at intermediate links, so that when the user is wearing the device he can control the full force interaction with the exoskeleton and guide/be guided with all articulations of the arm (wrist, shoulder, elbow). As depicted in figure 3(a), the exoskeleton has a serial architecture isomorphic with the human kinematics that comprises: a shoulder joint fixed in space and composed by three active joints $J_{1}, J_{2}$ and $J_{3}$; an active elbow joint $J_{4}$; and a passive revolute joint $J_{5}$ allowing for wrist prono/supination. For a more detailed description of both RehabExos and actuation groups, the reader can refer to [17].

The three joints $J_{1}, J_{2}$ and $J_{4}$ of the exoskeleton are motorized through identical actuation groups. Each joint features a custommade frameless brushless torque motor integrating a compact Harmonic Drive (HD) component set and a torque sensor consisting of two fully balanced strain gauge bridges placed on different beams of a thin planar sprocket hub, which is located at the joint output shaft (see figure 3(b)). The actuator provides a joint output torque equal to $150 \mathrm{Nm}$ with an overall weight equal to $3.7 \mathrm{Kg}$, motor shaft inertia reduced to the joint output shaft $\mathrm{Jm}=3.7 \mathrm{Kgm}^{2}$, overall joint torsional stiffness reduced to the joint output shaft $k=1.13 \mathrm{kNm} / \mathrm{rad}$. The Harmonic Drive performs a reduction equal to 100:1. Owing to the adopted mechanical components, the joint features limited backdrivability at motor power-off and limited mechanical complexity to ease maintenance as well as cost reduction. The internal joint-torque sensor introduces controlled torsional compliance that is used at the same time to transmit joint torque actuation from the motor to the link and to measure it. Joint $J_{3}$ is characterized by a tendon transmission that is used to transmit the actuation torque through an open semi-circular guide.
Despite further augmenting the actuation group compliance, the availability of joint-torque sensors enables for multicontact force control at multiple points distributed over the links and, additionally, makes it possible: 1) to close a stable high-bandwidth torque inner loop around each joint which is weakly affected by robot link variable inertia; 2) to suppress robot vibrations produced by the inherent transmission compliance (Harmonic Drive); 3) to reduce internal disturbance torques caused by actuator and reducer (for instance friction losses, actuator torque ripples and gear teeth wedging actions); to measure externally applied forces/moments and complex nonlinear dynamic interactions between joints and links.

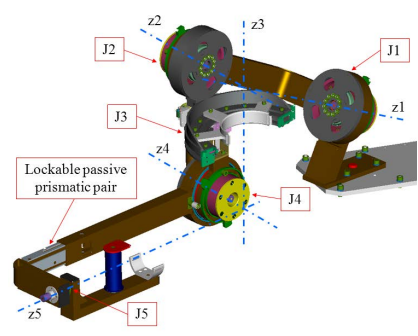

(a) Exoskeleton kinematics

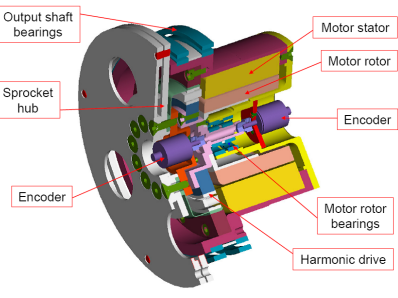

(b) CAD section of the joint actuator
Figure 3: The Rehab-Exos CAD model showing overall kinematics and actuator structure

\subsection{Control Hardware}

A decentralized control architecture was adopted that can guarantee both optimal signal to noise ratio in the acquisition of analogical signals, i.e. force sensors, and higher standards of safety.

For safety requirements a bus architecture was adopted based on EtherCAT ET1100, as shown in figure 4. EtherCAT is an Ethernet-Based Communication Industrial International Standard since 2007, supervised by ETG(EtherCAT Thecnology Group). It is provided with a complete set of protocols, mechanisms, development tools and electronic devices needed to carry out communication implementation in the field of industrial automation. The EtherCAT communication network consists of one master controller and four slave controllers Ethercat Slave Controller for each actuation joint. The master controller is handled by xPC Target, real-time Operating System Matlab toolbox, and is in charge of the executing the centralized control model $(500 \mu \mathrm{sec})$.

Motors of the exoskeleton consist of three 170VDC Power Supplied brushless motors on the 1st, 2nd and 4th joint each ones driven by programmable current driver and one 48VDC Power Supplied DC motor on the 3th joint. All of them are provided with one incremental encoder and one torque sensor.

Each Ethercat Slave Controller (ESC) board is a "PERCRO designed" control board consisting of an up-to $72 \mathrm{Mhz}$ ARM7 Microcontroller, with 4 14-bit DAC output interfaces, 10 14-bit Analogto-Digital Converter (ADC) channels, and the EtherCAT ET1100 controller linking to double-port Ethernet interface. Each ESC board can locally acquire data from encoders and force sensors. Each torque sensor is acquired through 2 Wheatstone full-bridge channels that are pre-amplified averaged and digitally converted with an A/D resolution conversion of 14 bits. Moreover each ESC performs estimation of speed and acceleration from position data.

\section{SINGLE JOINT MODEL}

Due to the elasticity of the harmonic drive speed reducer (for joints 1,2 and 4) and of tendon transmission for joint 3, each joint can be modeled as a 2-mass system. A single joint model is shown in figure 5. Using as notation $\theta_{m, i}$ and $\theta_{j, i}$ as the symbols for motor and joint angles respectively for joint $i$, and introducing two coefficients $k_{t, i}$ 


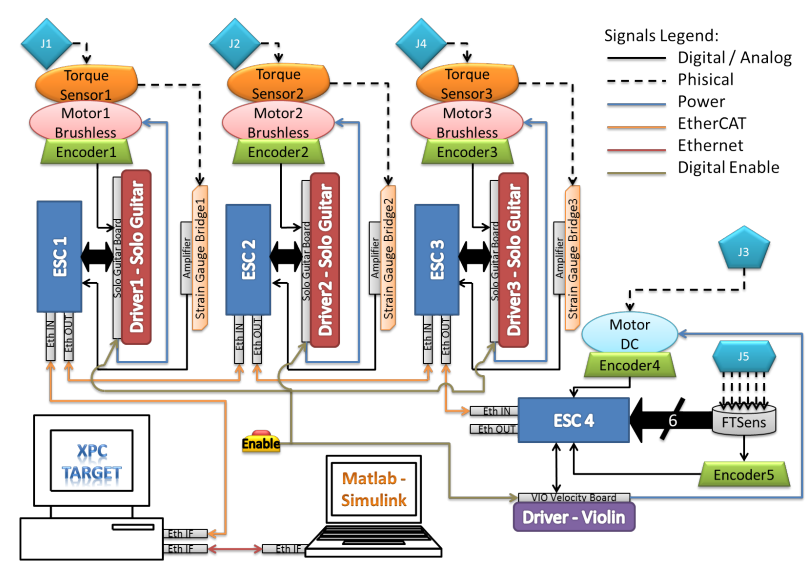

Figure 4: The decentralized control architecture

and $c_{t, i}$ to model the elasticity and viscosity of the transmission, that were experimentally characterized, the following equations can be written for single joint dynamics:

$$
\left\{\begin{array}{r}
J_{m, i} \ddot{\theta}_{m, i}+c_{m, i} \dot{\theta}_{m, i}+c_{t, i}\left(\dot{\theta}_{m, i}-\dot{\theta}_{j, i}\right)+k_{t, i}\left(\theta_{m, i}-\theta_{j, i}\right)= \\
\tau_{m, i}+\tau_{d, i} \\
J_{l, i} \ddot{\theta}_{j, i}+c_{t, i}\left(\dot{\theta}_{j, i}-\dot{\theta}_{m, i}\right)+k_{t, i}\left(\theta_{j, i}-\theta_{m, i}\right)= \\
\tau_{l, i}
\end{array}\right.
$$

where, referring to the the i-th joint, $J_{m, i}$ is motor inertia, $J_{l, i}$ is average link inertia considered as constant, $c_{m, i}$ is the viscous friction coefficient between motor rotor and stator, $\tau_{m, i}$ is the motor torque, $\tau_{d, i}$ is a disturbance torque acting on the motor rotor which accounts for internal friction and ripple effects of both motor and harmonic drive, while $\tau_{l, i}$ is the external torque acting directly on the output link which accounts for the exogenous input due to the interaction with the human, and endogenous input accounting for unmodeled non-linear effects, such as dynamic or gravity forces.

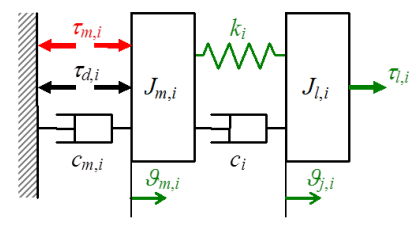

Figure 5: The 2-mass model for each joint

\subsection{Single joint acceleration estimation}

The full dynamics model of the exoskeleton, as described in section 4.1 , is obviously dependent of the acceleration of each joint. In order to estimate and compensate for the dynamics of the device, an observer for the joint acceleration has been designed. For each joint, the acceleration can be derived as indirect measurement from motor encoder $\theta_{m, i}$, joint torque $\tau_{s, i}$ and the imposed control torque $\tau_{m, i} . \tau_{s, i}$ is the torque measured by the sensor at the joint and can be expressed as in equation 11 .

In particular, since $\tau_{m, i}-\tau_{s, i}$ is the torque acting on the actuation group (motor plus gearhead) and the losses can be modeled as a static and a velocity-dependent viscous friction, the acceleration can be estimated as:

$$
\left\{\begin{array}{r}
\ddot{\theta}_{m, i}=0 \quad \text { for }-\tau_{A, i}<\tau_{m, i}-\tau_{s, i}<\tau_{A, i} \\
\ddot{\theta}_{m, i}=\frac{\tau_{m, i}-\tau_{s, i}-c_{m, i} \dot{\theta}_{m, i}}{J_{m, i}}
\end{array}\right.
$$

where $\tau_{A, i}$ is the static friction torque and $c_{m, i}$ is the dynamic friction coefficient. Both coefficient were experimentally evaluated. The torque saturation effects due to power supply voltage limits are modeled as:

$$
k_{c} \frac{-V_{\max }-k_{v} \dot{\theta}_{m, i}}{R}<\tau_{m, i}<k_{c} \frac{V_{\max }-k_{v} \dot{\theta}_{m, i}}{R}
$$

depending on the electric constants of each motor, and in particular where $k_{c}$ is the associated torque constant, $k_{v}$ is the velocity constant, $R$ is the winding terminal resistance and $V_{\max }$ is the maximum supply voltage to the motor. A diagram of the estimation of acceleration by control and measured torques is shown in figure 6 .

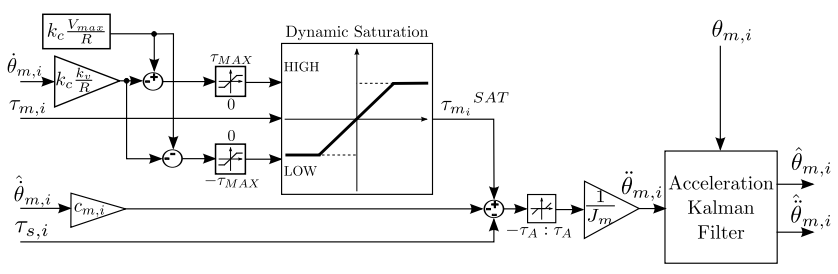

Figure 6: Estimation of the acceleration from torque measurement

While $\tau_{s, i}$ is directly measured by the joint torque sensor and $\tau_{m, i}$ is derived from motor command, an optimum Kalman observer has been used to estimate the acceleration term $\ddot{\theta}_{m, i}$ from direct position measurements.

The model can be expressed in the state variable form as follows:

$$
\left\{\begin{array}{r}
\dot{\mathbf{x}}=A \mathbf{x}+\Gamma d \\
\mathbf{y}=C \mathbf{x}
\end{array}\right.
$$

where

$\mathbf{x}=\left[\begin{array}{c}\theta_{m, i} \\ \dot{\theta}_{m, i} \\ \ddot{\theta}_{m, i}\end{array}\right] A=\left(\begin{array}{lll}0 & 1 & 0 \\ 0 & 0 & 1 \\ 0 & 0 & 0\end{array}\right) \Gamma=\left[\begin{array}{l}0 \\ 0 \\ 1\end{array}\right] C=\left(\begin{array}{lll}1 & 0 & 0 \\ 0 & 0 & 1\end{array}\right)$

and $d$ is the process noise.

The observer can be formulated as:

$$
\dot{\hat{\mathbf{x}}}=A \hat{\mathbf{x}}+L(\mathbf{y}-C \hat{\mathbf{x}})
$$

where $\mathrm{L}$ is the gain matrix of the observer. A scheme of the observer is depicted in figure (7).

As an example, the comparison between the real-time estimated acceleration (red dotted line) and the offline calculated acceleration (blue solid line) for the first two joints is shown in figure 8 .

\section{Centralized interaction torque control}

\subsection{Full dynamics model}

Based on the derived two-mass model of each joint, the full dynamic model of the exoskeleton can be derived and formulated in 


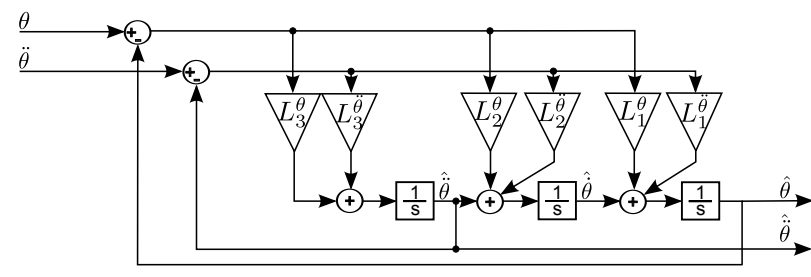

Figure 7: Block diagram of the acceleration observer
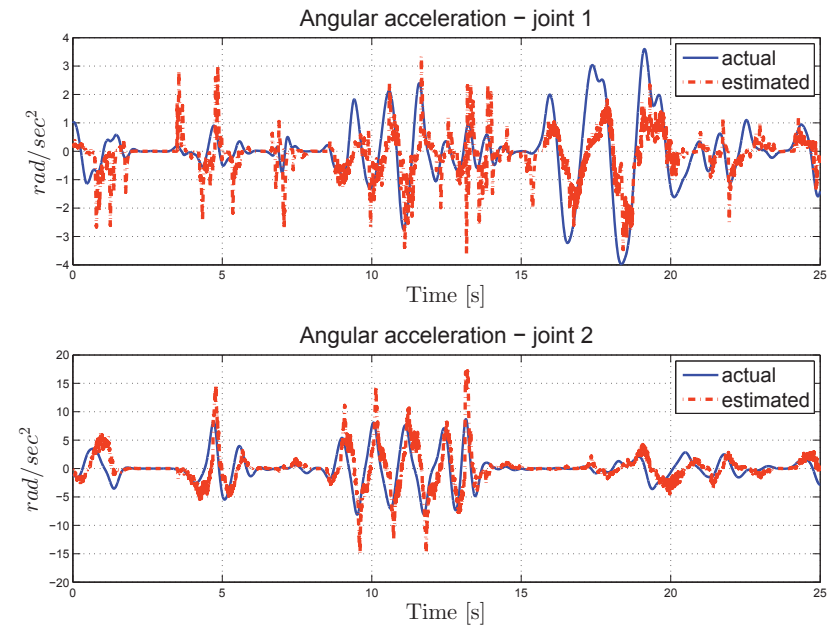

Figure 8: Comparison between the estimated and actual acceleration

matrix form as follows:

$$
\begin{aligned}
& J_{m} D \ddot{\boldsymbol{\theta}}_{m}+B_{m} D \dot{\boldsymbol{\theta}}_{\boldsymbol{m}}+C_{t}\left(D \dot{\boldsymbol{\theta}_{\boldsymbol{m}}}-\dot{\boldsymbol{\theta}}_{j}\right)+ \\
& +K_{t}\left(D \boldsymbol{\theta}_{\boldsymbol{m}}-\boldsymbol{\theta}_{j}\right)=\boldsymbol{\tau}_{\boldsymbol{m}}+\boldsymbol{\tau}_{\boldsymbol{d}} \\
& M\left(\boldsymbol{\theta}_{j}\right) \ddot{\boldsymbol{\theta}}_{\boldsymbol{j}}+C\left(\dot{\boldsymbol{\theta}}_{\boldsymbol{j}}, \boldsymbol{\theta}_{j}\right) \dot{\boldsymbol{\theta}}_{j}+C_{t}\left(\dot{\boldsymbol{\theta}_{j}}-D \dot{\boldsymbol{\theta}_{\boldsymbol{m}}}\right)+ \\
& +K_{t}\left(\boldsymbol{\theta}_{j}-D \boldsymbol{\theta}_{\boldsymbol{m}}\right)+G\left(\boldsymbol{\theta}_{j}\right)=J^{T} \mathbf{F}_{\mathbf{h}}
\end{aligned}
$$

where $D$ is a diagonal matrix modeling the reduction factor introduced by joint speed reducers, $J_{m}$ and $B_{m}$ are diagonal matrices modeling inertia and viscous friction at motor, $K_{t}$ and $C_{t}$ are respectively diagonal matrices modeling stiffness and damping associated to the elastic transmission, and $G$ represents the effects of gravity force on links. The external exogenous forces acting on the system are due to human interaction, represented by term $\mathbf{F}_{\mathbf{h}}$. It is important to note that we introduce cross-coupling among joints and non-linearities, with terms $C\left(\dot{\boldsymbol{\theta}}_{\boldsymbol{j}}, \boldsymbol{\theta}_{j}\right)$ modeling Coriolis effects and $M\left(\boldsymbol{\theta}_{\boldsymbol{j}}\right)$ representing link inertia.

Let us study the effect under static condition of the application of a motor torque that compensates for the non-linearity due to gravity, estimated as $\hat{G}\left(D \hat{\boldsymbol{\theta}}_{\boldsymbol{m}}\right)$, with:

$$
\boldsymbol{\tau}_{\boldsymbol{m}}=\hat{G}\left(D \hat{\boldsymbol{\theta}}_{\boldsymbol{m}}\right)+\mathbf{u}
$$

and with the assumption that $\mathbf{u}$ represents the actual control command. Under static conditions we find that:

$$
\mathbf{u}=-J^{T} \mathbf{F}_{\mathbf{h}}+G\left(\boldsymbol{\theta}_{j}\right)-\hat{G}\left(D \hat{\boldsymbol{\theta}}_{\boldsymbol{m}}\right)=-J^{T} \mathbf{F}_{\mathbf{h}}
$$

since $\hat{G}\left(D \hat{\theta}_{\mathbf{m}}\right) \simeq G\left(\boldsymbol{\theta}_{j}\right)$. Under dynamic conditions we can model the incomplete cancellation of the gravity component due to the transmission of actuation force through the elasticity of the speed reducer, by introducing a disturbance term $\delta \mathbf{g}=G\left(\boldsymbol{\theta}_{j}\right)-$ $\hat{G}\left(D \hat{\boldsymbol{\theta}}_{\boldsymbol{m}}\right)$, that can be summed up to $\mathbf{F}_{\mathbf{h}}$ as a disturbance noise supported by the operator. Moreover we have to consider that the real dynamics has terms $M\left(\boldsymbol{\theta}_{\boldsymbol{j}}\right)$ and $C\left(\dot{\boldsymbol{\theta}}_{\boldsymbol{j}}, \boldsymbol{\theta}_{j}\right)$ depending on the actual joint configuration. The first term can be decoupled into a diagonal constant component and a variation component as follows:

$$
M \ddot{\boldsymbol{\theta}}_{j}=\bar{M} \ddot{\boldsymbol{\theta}}_{\boldsymbol{j}}+\Delta M\left(\boldsymbol{\theta}_{j}\right) \ddot{\boldsymbol{\theta}}_{\boldsymbol{j}}
$$

So we can define a variable apparent dynamic force that we will call $\mathbf{F}_{\mathbf{d y n}}$ such that $J^{T} \Delta \mathbf{F}_{\mathbf{d y n}}\left(\dot{\boldsymbol{\theta}}_{\boldsymbol{j}}, \boldsymbol{\theta}_{j}\right)=-\Delta M\left(\boldsymbol{\theta}_{j}\right) \ddot{\boldsymbol{\theta}}_{\boldsymbol{j}}-$ $C\left(\dot{\boldsymbol{\theta}}_{\boldsymbol{j}}, \boldsymbol{\theta}_{j}\right) \dot{\boldsymbol{\theta}}_{\boldsymbol{j}}$ The new variable $\Delta \mathbf{F}_{\mathbf{d y n}}$, representing uncompensated and/or unmodeled dynamics, can be considered as disturbance force as well that will be counteracted by the human,

$$
\mathbf{F}_{\mathbf{l}}=\mathbf{F}_{\mathbf{h}}+\delta \mathbf{g}+\Delta \mathbf{F}_{\mathbf{d y n}}
$$

This in general states that the external forces are the sum of exogenous $\mathbf{F}_{\mathbf{h}}$ and endogenous inputs $\delta \mathbf{g}+\Delta \mathbf{F}_{\mathbf{d y n}}$. While exogenous inputs are unknown a priori and depending on human operator behavior, endogenous inputs can be estimated and compensated to some extent.

Introducing moreover the following variable substitution for joint torque $\tau_{s}$

$$
\left\{\begin{array}{l}
\boldsymbol{\tau}_{\boldsymbol{s}}=-K_{t}\left(D \boldsymbol{\theta}_{\boldsymbol{m}}-\boldsymbol{\theta}_{j}\right) \\
\dot{\boldsymbol{\tau}_{\boldsymbol{s}}}=-K_{t}\left(D \dot{\boldsymbol{\theta}_{\boldsymbol{m}}}-\dot{\boldsymbol{\theta}_{j}}\right) \\
\ddot{\boldsymbol{\tau}_{\boldsymbol{s}}}=-K_{t}\left(D \ddot{\boldsymbol{\theta}_{\boldsymbol{m}}}-\ddot{\boldsymbol{\theta}_{j}}\right)
\end{array}\right.
$$

dynamic equations can be reformulated as follows:

$$
\begin{aligned}
& J_{m} D \ddot{\boldsymbol{\theta}}_{\boldsymbol{m}}+B_{m} D \dot{\boldsymbol{\theta}}_{\boldsymbol{m}}=K_{t}^{-1} C_{t} \dot{\boldsymbol{\tau}_{\boldsymbol{s}}}+\boldsymbol{\tau}_{\boldsymbol{s}}+\mathbf{u}+\boldsymbol{\tau}_{\boldsymbol{d}} \\
& \bar{M} \ddot{\boldsymbol{\theta}}_{\boldsymbol{j}}+K_{t}^{-1} C_{t} \dot{\boldsymbol{\tau}_{\boldsymbol{s}}}+\boldsymbol{\tau}_{\boldsymbol{s}}=J^{T} \mathbf{F}_{\mathbf{l}}
\end{aligned}
$$

But we know that

$$
\begin{aligned}
& K_{t}^{-1} \ddot{\boldsymbol{\tau}}_{\boldsymbol{s}}+D \ddot{\theta}_{m}=\ddot{\theta}_{j} \\
& K_{t}^{-1} \dot{\boldsymbol{\tau}}_{\boldsymbol{s}}+D \dot{\theta}_{m}=\dot{\theta}_{j}
\end{aligned}
$$

Then making substitution of the above in (13) to eliminate $\boldsymbol{\theta}_{j}$ and its higher order derivatives, we obtain

$$
\bar{M} D \ddot{\boldsymbol{\theta}}_{m}+\bar{M} K_{t}^{-1} \ddot{\boldsymbol{\tau}}_{\boldsymbol{s}}+K_{t}^{-1} C_{t} \dot{\boldsymbol{\tau}}_{\boldsymbol{s}}+\boldsymbol{\tau}_{\boldsymbol{s}}=J^{T} \mathbf{F}_{\mathbf{l}}
$$

and then replacing $D \ddot{\theta}_{m}=J_{m}^{-1}\left\{-B_{m} D \dot{\boldsymbol{\theta}}_{\boldsymbol{m}}+K_{t}^{-1} C_{t} \dot{\boldsymbol{\tau}}_{\boldsymbol{s}}+\right.$ $\left.\boldsymbol{\tau}_{\boldsymbol{s}}+\mathbf{u}+\boldsymbol{\tau}_{\boldsymbol{d}}\right\}$ and defining $J_{i}^{-1}=\bar{M}^{-1}\left[I+\bar{M} J_{m}^{-1}\right]$, dynamic equations can be put in the following form:

$$
\left\{\begin{array}{c}
J_{m} D \ddot{\boldsymbol{\theta}}_{\boldsymbol{m}}+B_{m} D \dot{\boldsymbol{\theta}}_{\boldsymbol{m}}=K_{t}^{-1} C_{t} \dot{\boldsymbol{\tau}}_{\boldsymbol{s}}+\boldsymbol{\tau}_{\boldsymbol{s}}+\mathbf{u}+\boldsymbol{\tau}_{\boldsymbol{d}} \\
\ddot{\boldsymbol{\tau}}_{\boldsymbol{s}}+C_{t} J_{i}^{-1} \dot{\boldsymbol{\tau}}_{\boldsymbol{s}}+K_{t} J_{i}^{-1} \boldsymbol{\tau}_{\boldsymbol{s}}=K_{t} J_{m}^{-1} B_{m} D \dot{\boldsymbol{\theta}}_{m}+ \\
+\bar{M}^{-1} K_{t} J^{T} \mathbf{F}_{\mathbf{l}}-K_{t} J_{m}^{-1} \boldsymbol{\tau}_{\boldsymbol{d}}-K_{t} J_{m}^{-1} \mathbf{u}
\end{array}\right.
$$

This form of the dynamic equation is useful for defining a fullstate feedback control law and an optimal observer for the estimation of joint torque. 


\subsection{An optimal observer for estimation of joint torque}

A full-state feedback joint-torque controller for the exoskeleton requires the knowledge of the joint variables $\tau_{s, i}, \quad \dot{\tau}_{s, i}, \theta_{m, i}, \dot{\theta}_{m, i}, \tau_{d, i}, \tau_{l, i}$, where $\tau_{l}=J^{T} \mathbf{F}_{\mathbf{l}}$. Since each joint is only equipped with one digital incremental encoder measuring $\theta_{m, i}$ and one joint-torque sensor measuring $\tau_{s, i}$, a full-state Kalman filter has been designed to clean out both $\theta_{m, i}$ from quantization noise $w_{\theta, i}$ and $\tau_{s, i}$ from measurement noise $w_{\tau, i}$, as well as to estimate the remaining variables $\dot{\tau}_{s, i}, \dot{\theta}_{m, i}, \tau_{d, i}$ and $\tau_{l, i}$.

Based on the linear dynamic formulation, according to [18] we modeled the dynamics of $\tau_{d, i}$ and $\tau_{l, i}$ as two distinct Wiener processes (i.e. as two distinct non-stationary random processes) $\dot{\tau}_{d, i}=v_{d, i}$ and $\dot{\tau}_{l, i}=v_{l, i}$, which, in practice, it is equivalent to consider only forces $\tau_{d, i}$ and $\tau_{l, i}$ to be continuous and varying with independent increments. Then the following meta-system can be derived from equation (16):

$$
\left\{\begin{array}{r}
\dot{\tau}_{\mathbf{i}}=A_{i} \tau_{\mathbf{i}}+B_{i} \tau_{m, i}+\Gamma_{i} v_{i} \\
\mathbf{y}_{\mathbf{i}}=C_{i} \tau_{\mathbf{i}}+w_{i}
\end{array}\right.
$$

where $\tau_{i}^{T}=\left[\tau_{s, i} \dot{\tau}_{s, i} \theta_{m, i} \dot{\theta}_{m, i} \tau_{l, i} \tau_{d, i}\right]$ is the meta-state vector, $v_{i}^{T}=\left[v_{l, i} v_{d, i}\right]$ is the vector of process noises with variances $V_{l, i}$ and $V_{d, i}, w_{i}^{T}=\left[v_{\tau, i} v_{\theta, i}\right]$ is the vector of measurement noises with variances $W_{l, i}$ and $W_{d, i}$, whereas:

$$
\begin{gathered}
A=\left(\begin{array}{cccccc}
\frac{c_{t, i}}{J_{i}} & \frac{-k_{t, i} b_{m, i}}{J_{i}} & 0 & 0 & \frac{k_{t, i}}{J_{l, i}} & \frac{-k_{t, i}}{J_{m, i}} \\
1 & 0 & 0 & 0 & 0 & 0 \\
\frac{c_{t, i} k_{t, i}}{J_{m, i}} & \frac{1}{J_{m, i}} & \frac{-b_{m, i}}{J_{m, i}} & 0 & 0 & \frac{1}{J_{m, i}} \\
0 & 0 & 1 & 0 & 0 & 0 \\
0 & 0 & 0 & 0 & 0 & 0 \\
0 & 0 & 0 & 0 & 0 & 0
\end{array}\right) \\
B_{i}=\left[\begin{array}{c}
\frac{-k_{t, i}}{J_{m, i}} \\
0 \\
\frac{1}{J_{m, i}} \\
0 \\
0 \\
0
\end{array}\right] \quad \Gamma=\left(\begin{array}{cc}
0 & 0 \\
0 & 0 \\
0 & 0 \\
0 & 0 \\
1 & 0 \\
0 & 1
\end{array}\right) \quad C=\left(\begin{array}{cc}
0 & 0 \\
1 & 0 \\
0 & 0 \\
0 & 1 \\
0 & 0 \\
0 & 0
\end{array}\right)
\end{gathered}
$$

\section{FULL STATE FEEDBACK CONTROLLER}

Given the state observer defined in previous section, we can define the following state feedback control law:

$$
\mathbf{u}=B_{m} D \dot{\boldsymbol{\theta}}_{m}+J_{m} \bar{M}^{-1} J^{T} \mathbf{F}_{\mathbf{l}}-\tau_{\mathbf{d}}-J_{i}^{-1} J_{m} \boldsymbol{\tau}_{\boldsymbol{s}}^{D}+K_{p} \mathbf{e}+K_{d} \dot{\mathbf{e}}
$$

where $\mathbf{e}=\tau_{s}-\tau_{s}^{D}$ is the error on sensor torque, given the desired sensor torque $\tau_{s}^{D}$. Let us assume moreover that $\tau^{D}=0$ and $\ddot{\tau^{D}}=0$, so that $\dot{\mathrm{e}}=\dot{\tau}_{s}$ and $\ddot{\mathrm{e}}=\ddot{\tau}_{s}$

Inputs are given from the state observer (18). We find so that the modified dynamics with the control law (19), leads to a stable error dynamics equation:

$$
\left\{\begin{array}{r}
\ddot{\theta}_{\mathbf{m}}=\ddot{\theta}_{\mathbf{j}}-K_{t}^{-1} \ddot{\mathbf{e}} \\
0=\ddot{\mathbf{e}}+\left(C_{t} J_{i}^{-1}+K_{d} K_{t} J_{m}^{-1}\right) \dot{\mathbf{e}}+\left(K_{t} J_{i}^{-1}+K_{p} K_{t} J_{m}^{-1}\right) \mathbf{e}
\end{array}\right.
$$

The convergence of error e to zero can so be adjusted by choosing the proportional and derivative gains $K_{p}$ and $K_{d}$, to obtain the desired dynamic response.

\section{DYNAMICS COMPENSATION}

The torques measured by joint sensors are due to the human force and any load applied on the links $\left(\mathbf{F}_{1}\right)$. To have a good estimation of human forces by torque sensors, it is necessary to remove from torque measurements the gravity and dynamics loads applied to the links. The gravity contribution depends only on the pose of the exoskeleton, can be calculated by the position signals provided by the motor encoders, and is already compensated in feed-forward by the term $\hat{G}\left(D \hat{\boldsymbol{\theta}}_{\boldsymbol{m}}\right)$ in $\boldsymbol{\tau}_{\boldsymbol{m}}$, except for the term $\delta \mathbf{g}$. On the other side, the dynamics contribution depends both on the pose and the acceleration and velocity of the links, which are not directly provided by any sensor, but are provided in first approximation as $D \hat{\boldsymbol{\theta}_{m}}$ by the observer described in section 3.1.

The dynamics torques due to the links inertia measured by the joint torque sensors can be so estimated as follows:

$$
\hat{\boldsymbol{\tau}}_{\boldsymbol{d y n} \boldsymbol{n}} \bar{\sim}\left(D \boldsymbol{\theta}_{\boldsymbol{m}}\right) D \ddot{\boldsymbol{\theta}}_{\boldsymbol{m}}+\hat{C}\left(D \boldsymbol{\theta}_{\boldsymbol{m}}, D \dot{\boldsymbol{\theta}}_{\boldsymbol{m}}\right) D \dot{\boldsymbol{\theta}}_{\boldsymbol{m}}
$$

Both matrix $\hat{M}$ and $\hat{C}$ are calculated taking into account for each joint the inertia of the parts supported by the torque sensor, discarding the inertia of the actuator of the joint.

The compensation torques $\alpha \hat{\boldsymbol{\tau}}_{d y n}$, with $0<\alpha<1$, are a percentage of the estimated torques $\hat{\tau}_{d y n}$; they are added to the desired torques $\boldsymbol{\tau}_{\mathbf{s}}{ }^{\boldsymbol{D}}$ as input to the state feedback controller according to (19) and feed-back with the estimated torque $\hat{\tau}_{\mathbf{s}}$, according to the scheme of figure 9 .

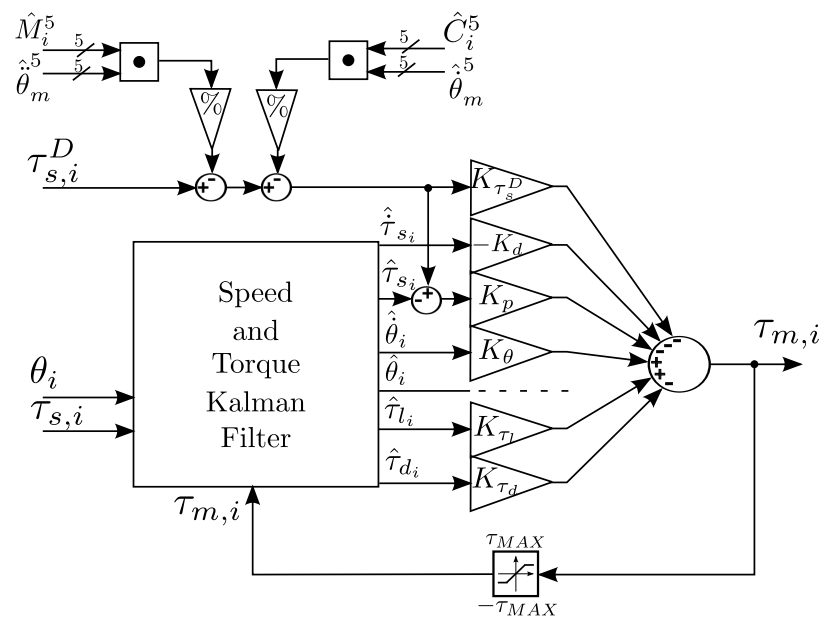

Figure 9: The full state control feedback

\subsection{Desired torque tracking}

In order to validate the interaction torque control and to evaluate the torque tracking performance, an additional force sensor has been mounted on the end effector of the exoskeleton to measure the actual forces $\mathbf{F}_{\mathbf{h}}^{*}$ that the user applies. The reflected torques at the joint are calculated as $\tau_{\mathbf{s}}^{*}=J^{T} \mathbf{F}_{\mathbf{h}}^{*}$ and then compared to the interaction torques estimated by the optimal observer. The experimental results for torque tracking, with a desired torque $\tau_{s}^{D}=0$, are shown for the second joint $J_{2}$ in figure 10 , that is the joint with the highest link inertia. A user has moved without constraints the exoskeleton, grabbing the force sensor mounted at the end effector. The control was set to follow his movement at zero-torque without (figure 10(a)) and with (figure 10(b)) dynamics compensation. The upper plots show the joint position (blue solid line) and acceleration (red dotted line), to demonstrate the movements were similar 
in both cases. The lower plots represent the interaction torques estimated by the observer (blue solid line) and measured by the force sensor (red dotted line). Even if the estimated torques are similar in both cases, with dynamics compensation the actual interaction forces are lower, demonstrating that torque tracking is more precise and the user has to compensate less for the links dynamics.
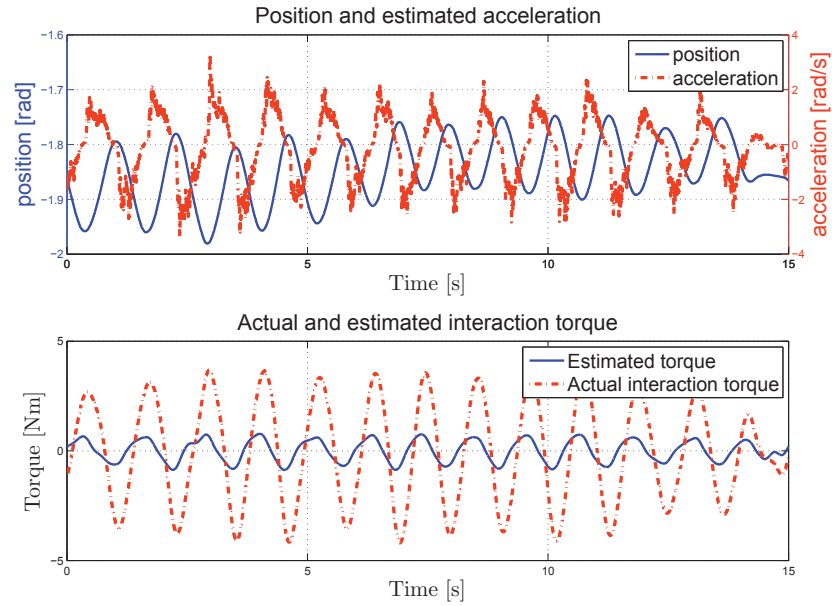

(a) Without dynamics compensation
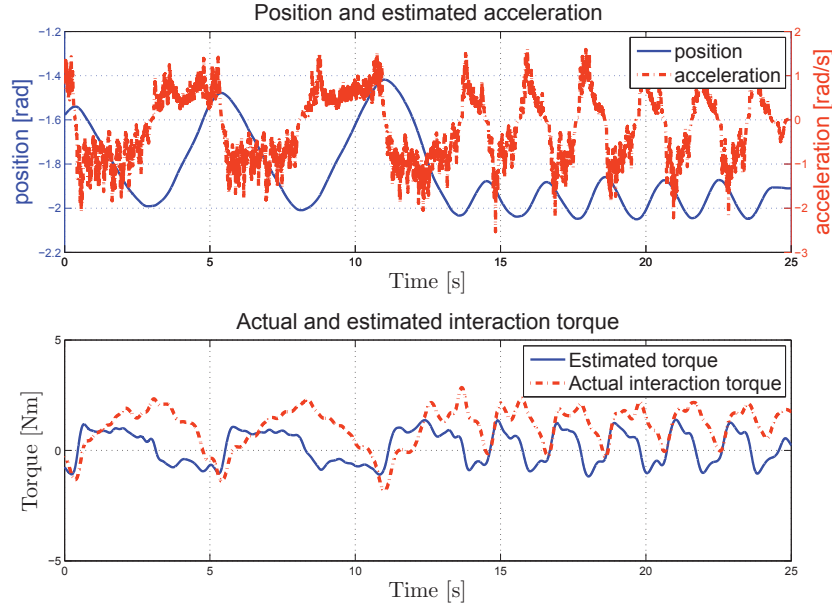

(b) With dynamics compensation

Figure 10: Comparison between the measured torque $\tau_{s}$ and calculated torque by force sensor

\section{HAPTIC RENDERING}

The implemented torque control can be used to render the interaction forces with a virtual surface with given stiffness and damping, acting as an impedance control. The contact force at the end effector is proportional to the penetration and the speed of penetration into the virtual object. The force is then converted to desired torques at the joints according to the following equation:

$$
\tau_{\mathbf{S}}^{\mathbf{D}}=J^{T} \mathbf{F}_{\mathbf{E E}}=J^{T}(K d-b \dot{d}) \mathbf{n}
$$

where $n$ is the normal to the surface, $d$ is the penetration in the object, $K$ is the simulated stiffness, $\dot{d}$ is the speed of penetration and $b$ is the simulated internal damping. The computed desired torques represents the input for the optimal torque observers of each joint, that assure the correct torque tracking. The design of the exoskeleton allows to control the torque on every joint, so it is possible to render an interaction force not only at the end effector but on every link of the robot. In this case, in equation (22) a modified Jacobian will be used to convert forces not applied on the end effector.

\subsection{Experimental test}

The performance of the impedance control has been assessed by experimental tests using the force sensor mounted on the end effector of the exoskeleton. The forces measured by the sensor are not used for the torque control, but only for acquiring the actual forces applied on the user hand. In the experiments the user grabs the exoskeleton only by the end effector, without applying any other force on the links. For experimental purposes we implemented the haptic rendering with a slanted flat surface with simulated stiffness $K=5000 \mathrm{~N} / \mathrm{m}$ and internal damping $k=300 \mathrm{Ns} / \mathrm{m}$.

Figure 11 shows the comparison between the desired force orthogonal to the virtual surface (red dotted line), calculated by the control system, the actual force measured by the sensor at the end effector (blue solid line) and the penetration into the virtual surface (black solid line).

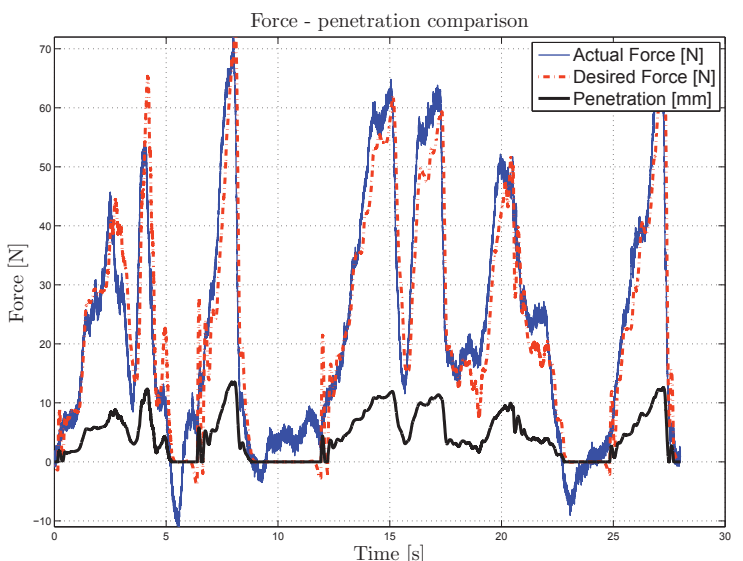

Figure 11: Comparison between the desired and actual force on the end effector

Figure 12 shows the interaction force with the virtual wall while sliding and pushing on the surface. The measured force is decomposed in the orthogonal component (black dotted line) and the tangential components (blue and red lines)

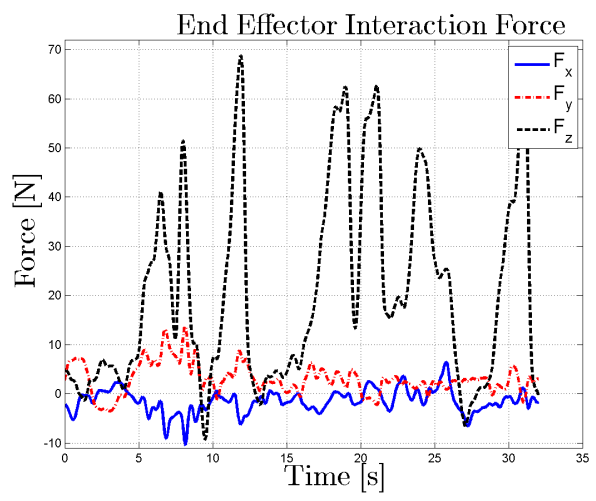

Figure 12: Orthogonal and tangential components of interaction force 


\section{Conclusion}

An interaction torque control of an exoskeleton with torque joint sensors has been developed and validated by experimental tests in haptic interaction tasks. The kinematics and dynamics of the device are calculated by a full dynamics model implemented in a centralized torque control. The torque tracking for each joint is performed by single-joint full-state Kalman filter and a torque feedback controller. The centralized control provides to each single-joint observer the desired torque for force feedback and an estimation of the joint torques due to links dynamic loads to be compensated by the control as feed-forward contributions. Experimental tests have been carried out to validate the fidelity of force feedback during haptic interaction with virtual objects and to assess the improvement in torque tracking adding partial compensation of the links dynamics. Results show how the presented approach is effective for estimating the human interaction force cleaned up of the inertial and gravity contributions due to the non negligible mechanical properties of the exoskeleton structure. This is reflected in better performance when high fidelity force rendering is required, such as in the case of rehabilitation applications.

\section{ACKNOWLEDGEMENTS}

This work has been partially funded from the EU FP7 project $n$. 257695 VERE and the EU FP7 project n. 601165 WEARHAP.

\section{REFERENCES}

[1] A. Albu-Schäffer, S. Haddadin, C. Ott, A. Stemmer, T. Wimböck, and G. Hirzinger. The dlr lightweight robot: design and control concepts for robots in human environments. Industrial Robot: An International Journal, 34(5):376-385, 2007.

[2] M. Bergamasco, A. Frisoli, and C. Avizzano. Exoskeletons as manmachine interface systems for teleoperation and interaction in virtual environments. Advances in Telerobotics, pages 61-76, 2007.

[3] C. Carignan, M. Liszka, and S. Roderick. Design of an arm exoskeleton with scapula motion for shoulder rehabilitation. In Advanced Robotics, 2005. ICAR'05. Proceedings., 12th International Conference on, pages 524-531. IEEE, 2005.

[4] C. R. Carignan and K. R. Cleary. Closed-loop force control for haptic simulation of virtual environments. Haptics-e, 1(2):1-14, 2000.

[5] C. R. Carignan, M. P. Naylor, and S. N. Roderick. Controlling shoulder impedance in a rehabilitation arm exoskeleton. In Robotics and Automation, 2008. ICRA 2008. IEEE International Conference on, pages 2453-2458. IEEE, 2008.

[6] A. Frisoli, F. Salsedo, M. Bergamasco, B. Rossi, and M. Carboncini. A force-feedback exoskeleton for upper-limb rehabilitation in virtual reality. Applied Bionics and Biomechanics, 6(2):115-126, 2009.

[7] P. Garrec, J. Friconneau, Y. Measson, and Y. Perrot. Able, an innovative transparent exoskeleton for the upper-limb. In Intelligent Robots and Systems, 2008. IROS 2008. IEEE/RSJ International Conference on, pages 1483-1488. IEEE, 2008.

[8] H. Kazerooni. Exoskeletons for human power augmentation. In Intelligent Robots and Systems, 2005.(IROS 2005). 2005 IEEE/RSJ International Conference on, pages 3459-3464. IEEE, 2005.

[9] J. Klein, S. Spencer, J. Allington, J. E. Bobrow, and D. J. Reinkensmeyer. Optimization of a parallel shoulder mechanism to achieve a high-force, low-mass, robotic-arm exoskeleton. Robotics, IEEE Transactions on, 26(4):710-715, 2010.

[10] H. S. Lo and S. Q. Xie. Exoskeleton robots for upper-limb rehabilitation: State of the art and future prospects. Medical engineering \& physics, 34(3):261-268, 2012.

[11] Y. Mao and S. K. Agrawal. Transition from mechanical arm to human arm with carex: A cable driven arm exoskeleton (carex) for neural rehabilitation. In Robotics and Automation (ICRA), 2012 IEEE International Conference on, pages 2457-2462. IEEE, 2012.

[12] S. Marcheschi, F. Salsedo, M. Fontana, and M. Bergamasco. Body extender: whole body exoskeleton for human power augmentation. In Robotics and Automation (ICRA), 2011 IEEE International Conference on, pages 611-616. IEEE, 2011.
[13] M. Mihelj, T. Nef, and R. Riener. Armin ii-7 dof rehabilitation robot: mechanics and kinematics. In Robotics and Automation, 2007 IEEE International Conference on, pages 4120-4125. IEEE, 2007.

[14] T. Nef, M. Mihelj, and R. Riener. Armin: a robot for patientcooperative arm therapy. Medical and Biological Engineering and Computing, 45(9):887-900, 2007.

[15] J. C. Perry, J. Rosen, and S. Burns. Upper-limb powered exoskeleton design. Mechatronics, IEEE/ASME Transactions on, 12(4):408-417, 2007.

[16] N. G. Tsagarakis and D. G. Caldwell. Development and control of a soft-actuated exoskeleton for use in physiotherapy and training. Autonomous Robots, 15(1):21-33, 2003.

[17] R. Vertechy, A. Frisoli, A. Dettori, M. Solazzi, and M. Bergamasco. Development of a new exoskeleton for upper limb rehabilitation. In Rehabilitation Robotics, 2009. ICORR 2009. IEEE International Conference on, pages 188-193. IEEE, 2009.

[18] R. Vertechy, A. Frisoli, M. Solazzi, D. Pellegrinetti, and M. Bergamasco. An interaction-torque controller for robotic exoskeletons with flexible joints: Preliminary experimental results. In Intelligent Robots and Systems (IROS), 2012 IEEE/RSJ International Conference on, pages 335-340. IEEE, 2012. 